\title{
Unravelling tidal dissipation in gaseous giant planets
}

\author{
M. Guenel ${ }^{1}$, S. Mathis ${ }^{1,2}$, and F. Remus ${ }^{3,1}$ \\ ${ }^{1}$ Laboratoire AIM Paris-Saclay, CEA/DSM - CNRS - Université Paris Diderot, IRFU/SAp Centre de Saclay, \\ 91191 Gif-sur-Yvette Cedex, France \\ e-mail: [mathieu.guenel; stephane.mathis]@cea.fr \\ 2 LESIA, Observatoire de Paris, CNRS UMR 8109, UPMC, Université Paris-Diderot, 5 place Jules Janssen, 92195 Meudon, France \\ 3 IMCCE, Observatoire de Paris, CNRS UMR 8028, UPMC, USTL, 77 avenue Denfert-Rochereau, 75014 Paris, France \\ e-mail: francoise.remus@obspm.fr
}

Received 16 April 2014 / Accepted 6 June 2014

\section{ABSTRACT}

\begin{abstract}
Context. Tidal dissipation in planetary interiors is one of the key physical mechanisms that drive the evolution of star-planet and planet-moon systems. New constraints on this dissipation are now obtained both in the solar and exo-planetary systems.

Aims. Tidal dissipation in planets is intrinsically related to their internal structure. Indeed, the dissipation behaves very differently when we compare its properties in solid and fluid planetary layers. Since planetary interiors consist of both types of regions, it is necessary to be able to assess and compare the respective intensity of the reservoir of dissipation in each type of layers. Therefore, in the case of giant planets, the respective contribution of the potential central dense rocky/icy core and of the deep convective fluid envelope must be computed as a function of the mass and the radius of the core. This will allow us to obtain their respective strengths. Methods. Using a method that evaluates the reservoir of dissipation associated to each region, which is a frequency-average of complex tidal Love numbers, we compared the respective contributions of the central core and of the fluid envelope.

Results. For Jupiter- and Saturn-like planets, we show that the viscoelastic dissipation in the core could dominate the turbulent friction acting on tidal inertial waves in the envelope. However, the fluid dissipation would not be negligible. This demonstrates that it is necessary to build complete models of tidal dissipation in planetary interiors from their deep interior to their surface without any arbitrary assumptions.

Conclusions. We demonstrate how important it is to carefully evaluate the respective strength of each type of dissipation mechanism in planetary interiors and to go beyond the usually adopted ad-hoc models. We confirm the significance of tidal dissipation in the potential dense core of gaseous giant planets.
\end{abstract}

Key words. hydrodynamics - waves - celestial mechanics - planets and satellites: interiors - planet-star interactions planets and satellites: dynamical evolution and stability

\section{Introduction and context}

The dissipation of tides is one of the key physical mechanisms that drive the evolution of planetary systems (Goldreich \& Soter 1966). At the same time, we understand little of the related dissipative processes acting both in rocky/icy and in fluid planetary layers even though they significantly affect the dynamics of star-planet and planet-moon systems (e.g. Efroimsky \& Lainey 2007; Auclair-Desrotour et al. 2014). Therefore, a strong effort must be undertaken to make realistic and reliable predictions for the dissipation rate of the kinetic energy of tidal displacements in planetary interiors. In this context, progress is achieved by using observational constraints in the solar and exoplanetary systems (e.g. Lainey et al. 2009; Husnoo et al. 2012; Albrecht et al. 2012). For example, tidal dissipation has been quantified for Jupiter and Saturn thanks to high-precision astrometric measurements as reported by Lainey et al. $(2009,2012)$ respectively. These works have demonstrated that these planets are likely to be the seat of strong dissipation, with in the case of Saturn at least a smooth dependence on the tidal excitation frequency. These results seem to favour the inelastic dissipation in their potential central dense rocky/icy core (e.g. Remus et al. 2012; Storch \& Lai 2014). However, the mass, the size, and the rheology of these cores are still unknown. Moreover, tides excite inertial waves in the deep fluid convective envelope. Their restoring force is the
Coriolis acceleration, and their dissipation by turbulent friction may be strong and therefore non-negligible (e.g. Ogilvie \& Lin 2004; Ogilvie 2013). As a result, it becomes necessary to explore and compare the reservoirs of tidal dissipation in each region with respect to their corresponding dissipative mechanism. This objective must be reached for all types of planets since they all potentially consist of a combination of solid and fluid layers.

In this first work, we focus on gaseous giant planets. By using simplified two-layer models as an exploratory tool for Jupiter- and Saturn-like planets, we apply the method described by Ogilvie (2013), which uses the frequency-dependent Love number to evaluate the reservoirs of dissipation both in their envelope and their core as a function of their mass and aspect ratios. This provides the first direct evaluation of the relative strength of the different mechanisms of tidal dissipation in a planet, which consists of different types of layers. In Sect. 2, we describe the main characteristics of our simplified planetary model. Next, we recall the method we used to compute the reservoirs of dissipation that is a result of viscoelastic dissipation in the core (Remus et al. 2012) and turbulent dissipation in the fluid envelope (Ogilvie 2013). In Sect. 3, we explore their respective strength for realistic values of the radius and the mass of the core, and we show why this approach is interesting. In conclusion, we discuss our results and the potential applications of this method. 


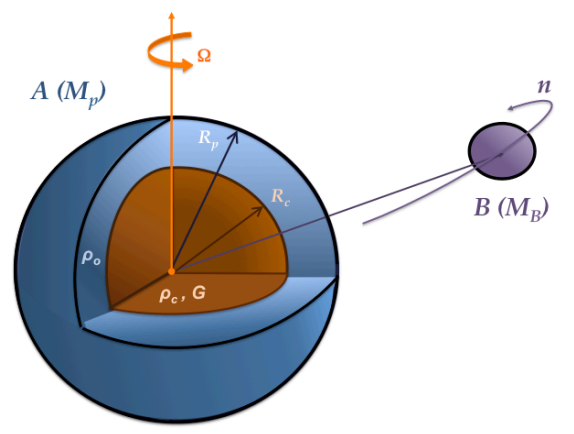

Fig. 1. Two-layer planet A of mass $M_{\mathrm{p}}$ and mean radius $R_{\mathrm{p}}$ and pointmass tidal perturber B of mass $M_{\mathrm{B}}$ orbiting with a mean motion $n$. The rocky/icy solid core of radius $R_{\mathrm{c}}$, density $\rho_{\mathrm{c}}$, and rigidity $G$ (see Eq. (4)) is surrounded by a convective fluid envelope of density $\rho_{o}$.

\section{Modelling tidal dissipation in gaseous giant planets}

\subsection{Two-layer model}

To study the respective contributions to the tidal dissipation of both the potential rocky/icy core and the fluid envelope of gaseous giant planets, we chose to adopt the simplified twolayer model used in Remus et al. (2012) and Ogilvie (2013, see Fig. 1). This model features a central planet A of mass $M_{\mathrm{p}}$ and mean radius $R_{\mathrm{p}}$ along with a point-mass tidal perturber $\mathrm{B}$ of mass $m$ orbiting with a mean motion $n$. Body A is assumed to be in moderate solid-body rotation with an angular velocity $\Omega$, so that $\epsilon^{2} \equiv \Omega^{2} / \sqrt{\mathcal{G} M_{\mathrm{p}} / R_{\mathrm{p}}^{3}} \ll 1^{1}$, where $\mathcal{G}$ is the gravitational constant. The rocky (or icy) solid core of radius $R_{\mathrm{c}}$ and density $\rho_{\mathrm{c}}$ is surrounded by a convective fluid envelope of density $\rho_{o}$. Both are assumed to be homogeneous for the sake of simplicity.

\subsection{Evaluating the tidal dissipation reservoirs}

The Love numbers quantify the response to the tidal perturbation induced on A by the companion B. More precisely, the Love number $k_{l}^{m}$, associated with the $(l, m)$ component of the time-dependent tidal potential $U$ that corresponds to the spherical harmonic $Y_{l}^{m}$, measures the ratio of the tidal perturbation of its self-gravity potential over the tidal potential at the surface of body A $\left(r=R_{\mathrm{p}}\right)$. These numbers are real for perfectly elastic or non-viscous layers, and in general depend on the tidal frequency $\omega=s n-m \Omega$ (with $s \in \boldsymbol{Z}$ ) (e.g. Efroimsky 2012; Remus et al. 2012) just like in any forced oscillating system. However, they are complex quantities in realistic planetary interiors where dissipation occurs, with a real part that accounts for the energy stored in the tidal perturbation, while the imaginary part accounts for the energy losses. Note that $\operatorname{Im}\left[k_{l}^{m}(\omega)\right]$ is proportional to $\operatorname{sgn}(\omega)$.

This imaginary part can be expressed in terms of the quality factor $Q_{l}^{m}(\omega)$, or equivalently, the tidal angle $\delta_{l}^{m}(\omega)$, which both depend on the tidal frequency

$Q_{l}^{m}(\omega)^{-1}=\sin \left[2 \delta_{l}^{m}(\omega)\right]=\operatorname{sgn}(\omega)\left|k_{l}^{m}(\omega)\right|^{-1} \operatorname{Im}\left[k_{l}^{m}(\omega)\right]$.

Then, following Ogilvie (2013), we calculate a weighted frequency-average of the imaginary part of the second-

\footnotetext{
1 In this regime, the Coriolis acceleration, which scales as $\Omega$, is taken into account, while the centrifugal acceleration, which scales as $\Omega^{2}$ is neglected.
}

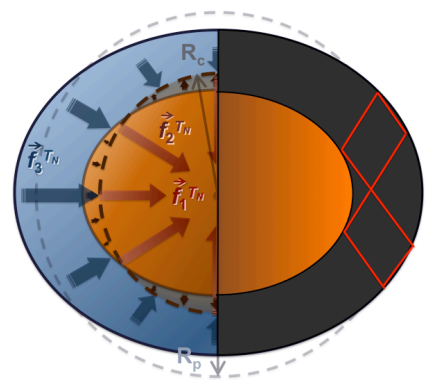

Fig. 2. Mechanisms of tidal dissipation in our two-layer planetary model: the inelastic dissipation in the dense rocky/icy core (left) and the dissipation due to the tidal inertial waves that reflect onto the core in the fluid convective envelope (right).

order Love number $k_{2}^{2}$, which we call the tidal dissipation reservoir:

$$
\int_{-\infty}^{+\infty} \operatorname{Im}\left[k_{2}^{2}(\omega)\right] \frac{\mathrm{d} \omega}{\omega}=\int_{-\infty}^{+\infty} \frac{\left|k_{2}^{2}(\omega)\right|}{Q_{2}^{2}(\omega)} \frac{\mathrm{d} \omega}{\omega} .
$$

This quantity can be defined for any values of $(l, m)$, but we here chose to consider the simplest case of a coplanar system for which the tidal potential $(U)$ reduces to the component $(2,2)$ as well as the quadrupolar response of A.

We now examine the two possible mechanisms of dissipation (see Fig. 2):

- in Sect. 2.3, we consider the dissipation associated to the inelasticity of the rocky/icy core following Remus et al. (2012);

- in Sect. 2.4, we focus on the dissipation of tidally excited inertial waves by the turbulent friction in the deep gaseous convective envelope following Ogilvie (2013). The integral in Eq. (2) then reduces to $\omega \in[-2 \Omega, 2 \Omega]$ because higherfrequency acoustic waves are filtered out.

\subsection{Inelastic dissipation in the core}

The inelastic tidal dissipation in the solid core is the result of its internal viscosity $(\eta)$. For the studied two-layer model, it is modified by the set of mechanical constraints, namely the gravitational forces $\left(f_{1}^{T_{N}}\right)$, the loading of the core due to its deformation $\left(f_{2}^{T_{N}}\right)$, and the hydrostatic pressure exerted by the surrounding fluid envelope $\left(\boldsymbol{f}_{3}^{T_{N}}\right)$, which is here assumed to be static and non-dissipative (see Dermott 1979; Remus et al. 2012, for a complete discussion and Fig. 2).

Following Remus et al. (2012) and Remus (2013), the second-order Love number $k_{2}^{2}(\omega)$ is given by

$k_{2}^{2}(\omega)=\frac{\tilde{H}+\alpha+3}{\frac{2}{3} \alpha \tilde{H}-\frac{3}{2}}$,

where $\alpha$ and $\tilde{H}$ are functions of the aspect ratio $\left(R_{\mathrm{c}} / R_{\mathrm{p}}\right)$, the density ratio $\left(\rho_{o} / \rho_{\mathrm{c}}\right)$, and the complex effective shear modulus $\hat{\mu}$ of the core:

$\alpha=1+\frac{5}{2} \frac{\rho_{\mathrm{c}}}{\rho_{o}}\left(\frac{R_{\mathrm{c}}}{R_{\mathrm{p}}}\right)^{3}\left(1-\frac{\rho_{o}}{\rho_{\mathrm{c}}}\right)$,

$\tilde{H}=\beta\left[\left(1+\frac{3}{2} \frac{\rho_{o}}{\rho_{\mathrm{c}}}\right)\left(1-\frac{\rho_{o}}{\rho_{\mathrm{c}}}\right)+\hat{\mu}(\omega)\right]$,

$\beta=\left(\frac{R_{\mathrm{c}}}{R_{\mathrm{p}}}\right)^{-5}\left(1-\frac{\rho_{o}}{\rho_{\mathrm{c}}}\right)^{-2}, \quad \frac{\hat{\mu}(\omega)}{\bar{\mu}(\omega)}=\gamma=\frac{19}{2 \rho_{\mathrm{c}} g_{\mathrm{c}} R_{\mathrm{c}}}$, 
where $\bar{\mu}$ is the complex shear modulus and $g_{\mathrm{c}}$ is the gravity at $r=R_{\mathrm{c}}$. Note also that $\operatorname{Im}\left[k_{2}^{2}(\omega)\right]$ scales as $\left(R_{\mathrm{c}} / R_{\mathrm{p}}\right)^{5}$ as $R_{\mathrm{c}} / R_{\mathrm{p}} \rightarrow$ 0 . This result is valid for any linear rheology, but the mechanical behaviour of the dense central rocky/icy cores in gaseous giant planets is poorly constrained (see e.g. Henning et al. 2009). For that reason, we used the simplest linear viscoelastic Maxwell model for which

$\operatorname{Re}[\bar{\mu}(\omega)]=\frac{\eta^{2} G \omega^{2}}{G^{2}+\eta^{2} \omega^{2}} \quad$ and $\quad \operatorname{Im}[\bar{\mu}(\omega)]=\frac{\eta G^{2} \omega}{G^{2}+\eta^{2} \omega^{2}}$

where $G$ is the rigidity and $\eta$ is the viscosity (see Henning et al. 2009; Remus et al. 2012). For this model, the core behaves as a rigid body when $\omega \gg \omega_{M}$, and as a fluid body when $\omega \ll \omega_{M}$, where $\omega_{M}=G / \eta$ is the Maxwell frequency. We find that

$\int_{-\infty}^{+\infty} \operatorname{Im}\left[k_{2}^{2}(\omega)\right] \frac{\mathrm{d} \omega}{\omega}=\frac{\pi G(3+2 \alpha)^{2} \beta \gamma}{\delta(6 \delta+4 \alpha \beta \gamma G)}$,

with $\delta=\left[\frac{2}{3} \alpha \beta\left(1-\frac{\rho_{o}}{\rho_{\mathrm{c}}}\right)\left(1+\frac{3}{2} \frac{\rho_{o}}{\rho_{\mathrm{c}}}\right)-\frac{3}{2}\right]$,

which is remarkably independent of the viscosity $\eta$ and vanishes for low values of $G$.

\subsection{Dissipation of inertial waves in the envelope}

Tidal dissipation in the fluid convective envelope of A originates from the excitation by $\mathrm{B}$ of inertial waves, which are driven by the Coriolis acceleration. They are damped by the turbulent friction, which can be modelled using a turbulent viscosity (Ogilvie $\&$ Lesur 2012). Its evaluation in our two-layer model was conducted by Ogilvie (2013), who assumed an homogeneous and perfectly rigid solid core without inelastic dissipation, while the envelope is homogenous and incompressible. The solutions of the system of dynamical equations for the fluid envelope written in the co-rotating frame are separated into a non-wavelike part (with subscripts ${ }_{n w}$ ), which corresponds to the immediate hydrostatic adjustment to the external tidal potential $(U)$, and a wavelike part (with subscript ${ }_{w}$ ) driven by the action of the Coriolis acceleration on the non-wavelike part:

$$
\left\{\begin{array} { l } 
{ \ddot { \boldsymbol { s } } _ { \mathrm { nw } } = - \nabla W _ { \mathrm { nw } } , } \\
{ h _ { \mathrm { nw } } ^ { \prime } + \Phi _ { \mathrm { nw } } ^ { \prime } + U = 0 , } \\
{ \rho _ { \mathrm { nw } } ^ { \prime } = - \nabla \cdot ( \rho \boldsymbol { s } _ { \mathrm { nw } } ) , } \\
{ \nabla ^ { 2 } \Phi _ { \mathrm { nw } } ^ { \prime } = 4 \pi \mathcal { G } \rho _ { \mathrm { nw } } ^ { \prime } , }
\end{array} \quad \text { and } \quad \left\{\begin{array}{l}
\ddot{\boldsymbol{s}}_{\mathrm{w}}+2 \Omega \boldsymbol{e}_{z} \times \dot{\boldsymbol{s}}_{\mathrm{w}}=-\nabla W_{\mathrm{w}}+\boldsymbol{f}, \\
h_{\mathrm{w}}^{\prime}=\Phi_{\mathrm{w}}^{\prime}=\rho_{\mathrm{w}}^{\prime}=0, \\
\nabla \cdot\left(\rho \boldsymbol{s}_{\mathrm{w}}\right)=0,
\end{array}\right.\right.
$$

where $\boldsymbol{s}$ is the displacement, $\boldsymbol{e}_{z}$ the unit vector along the rotation axis, $h$ the specific enthalpy, $\Phi$ the self-gravitational potential of $\mathrm{A}$, and $\rho$ is the density. Primed variables denote an Eulerian perturbation in relation to the unperturbed state with unprimed variables. Note that $U$ and $s$ are actually perturbations as well. Finally, $W \equiv W_{\mathrm{nw}}+W_{\mathrm{w}}=h^{\prime}+\Phi^{\prime}+U$, while $\boldsymbol{f}=-2 \Omega \boldsymbol{e}_{z} \times \dot{\boldsymbol{s}}_{\mathrm{nw}}$ is the acceleration driving the wavelike part of the solution.

The kinetic energy of the wavelike part of the solution can be derived without solving the whole system of equations, thanks to an impulsive calculation. This kinetic energy will eventually be dissipated (regardless of the exact physical processes at stake here, even if we know that it can be modelled by a turbulent viscosity) and is related to the tidal dissipation reservoir introduced

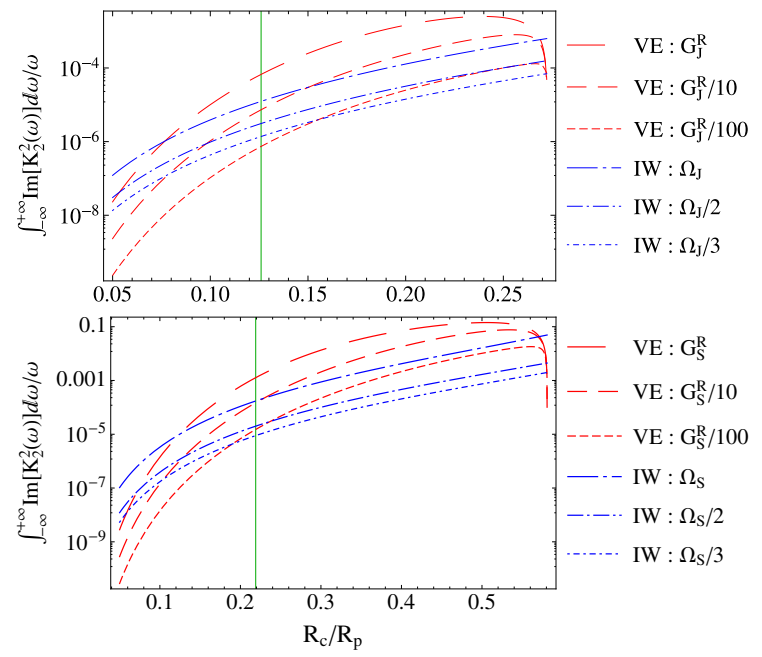

Fig. 3. Dissipation reservoirs for the viscoelastic dissipation in the core (red curves) and the turbulent friction in the fluid envelope (blue curves) in Jupiter- (above) and Saturn-like planets (below) as a function of the aspect ratio $R_{\mathrm{c}} / R_{\mathrm{p}}$, the rotation rate $\Omega$, and the rigidity of the core $G$, with fixed $R_{\mathrm{p}}$ and $M_{\mathrm{p}}$. We use the values $M_{\mathrm{c}} / M_{\mathrm{p}}=\{0.02,0.196\}$ from Guillot (1999) and Hubbard et al. (2009) for Jupiter and Saturn, respectively. The vertical green line corresponds to $R_{\mathrm{c}} / R_{\mathrm{p}}=\{0.126,0.219\}$.

in Eq. (2). The final result is (Ogilvie 2013)

$$
\begin{aligned}
& \int_{-\infty}^{+\infty} \operatorname{Im}\left[k_{2}^{2}(\omega)\right] \frac{\mathrm{d} \omega}{\omega}=\frac{100 \pi}{63} \epsilon^{2} \frac{\left(R_{\mathrm{c}} / R_{\mathrm{p}}\right)^{5}}{1-\left(R_{\mathrm{c}} / R_{\mathrm{p}}\right)^{5}} \\
& \quad \times\left[1+\frac{1-\rho_{o} / \rho_{\mathrm{c}}}{\rho_{o} / \rho_{\mathrm{c}}}\left(R_{\mathrm{c}} / R_{\mathrm{p}}\right)^{3}\right]\left[1+\frac{5}{2} \frac{1-\rho_{o} / \rho_{\mathrm{c}}}{\rho_{o} / \rho_{\mathrm{c}}}\left(R_{\mathrm{c}} / R_{\mathrm{p}}\right)^{3}\right]^{-2}
\end{aligned}
$$

\section{Comparison of the two dissipation mechanisms}

Our goal is to quantitatively compare the respective strength of the two dissipation mechanisms to determine if and when either one of them can be neglected in gaseous giant planets similar to Jupiter and Saturn. Their respective mass and radius are $M_{\mathrm{p}}=\{317.83,95.16\} M_{\oplus}$ and $R_{\mathrm{p}}=\{10.97,9.14\} R_{\oplus}$ with $M_{\oplus}=5.97 \times 10^{24} \mathrm{~kg}$ and $R_{\oplus}=6.37 \times 10^{3} \mathrm{~km}$ being the Earth's mass and radius. Their rotation rate are $\Omega_{\{\mathrm{J}, \mathrm{S}\}}=\left\{1.76 \times 10^{-4}, 1.63 \times 10^{-4}\right\} \mathrm{s}^{-1}$. Internal structure models for these bodies are still only poorly constrained. This is why we chose to explore wide ranges of core radii in Fig. 3 (covering the values considered possible by Guillot 1999, for Jupiter; and Hubbard et al. 2009, for Saturn) and core masses in Fig. 4 (covering the values considered possible by Guillot 1999; Nettelmann 2011; and Nettelmann et al. 2013). To do this, we used fixed values for the mass ratios $M_{\mathrm{c}} / M_{\mathrm{p}}$ (in Fig. 3) or for the aspect ratios $R_{\mathrm{c}} / R_{\mathrm{p}}$ (in Fig. 4), along with specific values of the angular velocity, $\Omega$, for tidal inertial waves (Eq. (7)) and of the rigidity $G$ (Eq. (5)) for the viscoelastic model. We chose to use as a reference $G_{\{\mathrm{J}, \mathrm{S}\}}^{\mathrm{R}}=\left\{4.46 \times 10^{10}, 1.49 \times 10^{11}\right\} \mathrm{Pa}$ which allows this dissipation model to match the dissipation measured by Lainey et al. (2009, 2012) in Jupiter at the tidal frequency of Io and in Saturn at the frequency of Enceladus (with $\left.\eta_{\{\mathrm{J}, \mathrm{S}\}}=\left\{1.45 \times 10^{14}, 5.57 \times 10^{14}\right\} \mathrm{Pa} \cdot \mathrm{s}\right)$. We assumed the core masses proposed by Guillot (1999) and Hubbard et al. (2009), that is $M_{\mathrm{c}}=\{6.41,18.65\} M_{\oplus}$, which yields the smallest core radii of $R_{\mathrm{c}}=\{0.126,0.219\} R_{\mathrm{p}}$. This allows us to avoid underestimating the solid dissipation reservoir that a poor choice of parameters could cause (see Fig. 9 in Remus et al. 2012). 

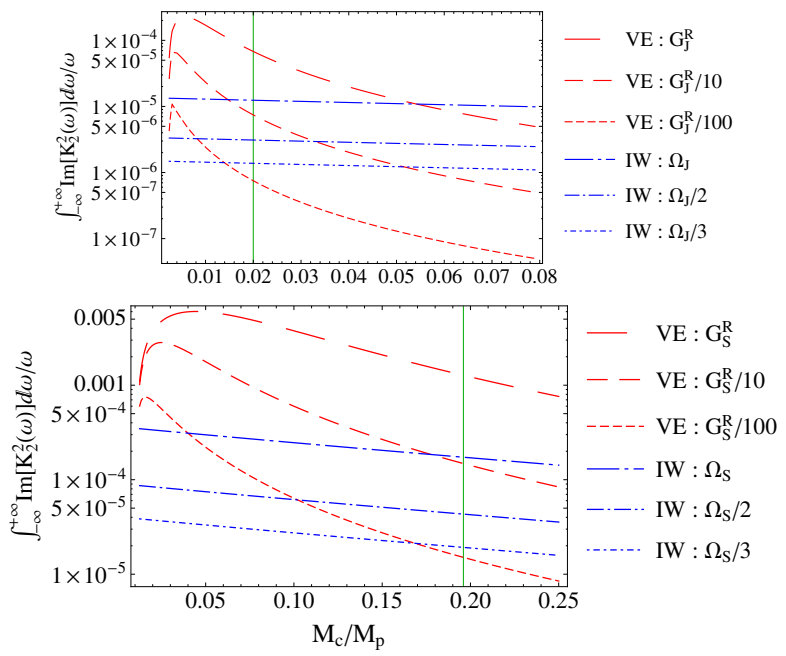

Fig. 4. Same as Fig. 3, but as a function of the mass ratio $M_{\mathrm{c}} / M_{\mathrm{p}}$ with fixed $M_{\mathrm{p}}$ and $R_{\mathrm{p}}$. We adopt $R_{\mathrm{c}} / R_{\mathrm{p}}=\{0.126,0.219\}$ for Jupiter and Saturn. The wide $M_{\mathrm{c}}$-ranges [1,3-25] $M_{\oplus}$ for Jupiter and [2-24] $M_{\oplus}$ for Saturn are those considered as possible by Guillot (1999), Nettelmann (2011), Nettelmann et al. (2013). The vertical green line corresponds to $M_{\mathrm{c}} / M_{\mathrm{p}}=\{0.02,0.196\}$.

\subsection{As a function of the core radius}

Figure 3 shows that for both dissipation models and both planets, the tidal dissipation reservoirs generally increase with the core radius until a critical value is reached, where $\rho_{o} / \rho_{\mathrm{c}}=1$, which is a singularity of the model; the density ratio decreases with the core radius since $R_{\mathrm{p}}$ and $M_{\mathrm{c}} / M_{\mathrm{p}}$ are fixed. Here, we adopted the values $M_{\mathrm{c}} / M_{\mathrm{p}}=\{0.02,0.196\}$ given by Guillot (1999) and Hubbard et al. (2009) respectively for Jupiter and Saturn. This result agrees with the predictions of Remus et al. (2012) for the core and of Ogilvie \& Lin (2004), Goodman \& Lackner (2009), Rieutord \& Valdettaro (2010), and Ogilvie (2013), who explained that inertial waves in a fluid spherical shell experience multiple reflections on its boundaries and follow specific paths called attractors, where shear layers occur, leading to a higher viscous dissipation than for a full sphere $(\mathrm{Wu}$ 2005, and Fig. 2). These plots show that in Jupiter- and Saturnlike gaseous giant planets, the two distinct mechanisms exposed in Sect. 2 can both contribute to tidal dissipation, and that therefore none of them can be neglected in general. Moreover, when $R_{\mathrm{c}} / R_{\mathrm{p}}>\{0.126,0.219\}, \Omega=\Omega_{\{\mathrm{J}, \mathrm{S}\}}$, and $G=G_{\{\mathrm{J}, \mathrm{S}\}}^{\mathrm{R}}$, the viscoelastic dissipation slightly dominates that in the fluid envelope until the singularity of the model is reached.

\subsection{As a function of the core mass}

If we now study the problem as a function of the core mass, we observe that the two tidal dissipation reservoirs associated with each model slightly decrease because the density ratio $\rho_{o} / \rho_{\mathrm{c}}$ decreases since $M_{\mathrm{p}}$ and $R_{\mathrm{c}} / R_{\mathrm{p}}$ are fixed. Here, we adopted $R_{\mathrm{c}} / R_{\mathrm{p}}=\{0.126,0.219\}$ for Jupiter and Saturn following previous sections. Again, the order of magnitude of each dissipation reservoir can be similar and there is no clear indication that either one of them is negligible. For $M_{\mathrm{c}} / M_{\mathrm{p}}=\{0.02,0.196\}, \Omega=\Omega_{\{\mathrm{J}, \mathrm{S}\}}$, and $G=G_{\{\mathrm{J}, S\}}^{\mathrm{R}}$, the viscoelastic dissipation slightly dominates that in the fluid envelope.

\section{Conclusions and perspectives}

We computed for the first time a direct comparison of the relative strength of tidal dissipation mechanisms in the interiors of gaseous giant planets. Even if it is necessary to keep in mind that this quantitative comparison was obtained using simplified twolayer planetary models, we are confident that this approach is reliable enough to explore and to evaluate the amplitude of both solid and fluid tidal dissipations and to compare them. In this framework, we find that to be able to reproduce the observed values of the tidal dissipation in Jupiter and in Saturn obtained thanks to high-precision astrometry (Lainey et al. 2009, 2012), we are in a situation where the viscoelastic dissipation in the core may dominate the turbulent friction acting on tidal inertial waves in the envelope. However, the fluid mechanism is not negligible, which shows that it is necessary to compute models that take into account all the possible dissipation mechanisms for complex planetary interiors. The action of each of them on the spins of bodies that constitute planetary systems and on their orbital architecture would be unveiled thanks to their behaviour as a function of the excitation frequency (Auclair-Desrotour et al. 2014) and of realistic formation/evolution simulations (e.g. Charnoz et al. 2011; Laskar et al. 2012). Moreover, this method which uses frequency-dependent complex Love numbers can be applied in the near future to realistically stratified solid and fluid regions (e.g. Ogilvie \& Lin 2004; Tobie et al. 2005) and to other types of planets such as icy giant planets and super-Earths, which are also composed of a superposition of both solid and fluid regions.

To derive reliable predictions from an ab-initio treatment of the mechanisms of tidal dissipation, it would be also necessary to simultaneously improve our understanding of the rheological behaviour of rocky and icy planetary layers and to take into account possible stable stratification, differential rotation, magnetic fields, and non-linear processes such as instabilities and turbulence in fluid regions.

Acknowledgements. We thank the referee, whose comments allowed us to improve the manuscript. M. Guenel was supported by CEA. This work was funded partly by the Programme National de Planétologie (CNRS/INSU), the Campus Spatial de l'Université Paris Diderot, the "L'Oréal-Académie des SciencesUnesco" fundation, and the Emergence-UPMC project EME0911 Encelade.

\section{References}

Albrecht, S., Winn, J. N., Johnson, J. A., et al. 2012, ApJ, 757, 18 Auclair-Desrotour, P., Le Poncin-Lafitte, C., \& Mathis, S. 2014, A\&A, 561, L7 Charnoz, S., Crida, A., Castillo-Rogez, J. C., et al. 2011, Icarus, 216, 535 Dermott, S. F. 1979, Icarus, 37, 310

Efroimsky, M. 2012, ApJ, 746, 150

Efroimsky, M., \& Lainey, V. 2007, J. Geophys. Res. (Planets), 112, 12003

Goldreich, P., \& Soter, S. 1966, Icarus, 5, 375

Goodman, J., \& Lackner, C. 2009, ApJ, 696, 2054

Guillot, T. 1999, Planet. Space Sci., 47, 1183

Henning, W. G., O’Connell, R. J., \& Sasselov, D. D. 2009, ApJ, 707, 1000

Hubbard, W. B., Dougherty, M. K., Gautier, D., \& Jacobson, R. 2009, The Interior of Saturn, eds. M. K. Dougherty, L. W. Esposito, \& S. M. Krimigis, 75

Husnoo, N., Pont, F., Mazeh, T., et al. 2012, MNRAS, 422, 3151

Lainey, V., Arlot, J.-E., Karatekin, Ö., \& van Hoolst, T. 2009, Nature, 459, 957

Lainey, V., Karatekin, Ö., Desmars, J., et al. 2012, ApJ, 752, 14

Laskar, J., Boué, G., \& Correia, A. C. M. 2012, A\&A, 538, A105

Nettelmann, N. 2011, Ap\&SS, 336, 47

Nettelmann, N., Püstow, R., \& Redmer, R. 2013, Icarus, 225, 548

Ogilvie, G. I. 2013, MNRAS, 429, 613

Ogilvie, G. I., \& Lesur, G. 2012, MNRAS, 422, 1975

Ogilvie, G. I., \& Lin, D. N. C. 2004, ApJ, 610, 477

Remus, F. 2013, Ph.D. Thesis, Univesité Pierre et Marie Curie

Remus, F., Mathis, S., Zahn, J.-P., \& Lainey, V. 2012, A\&A, 541, A165

Rieutord, M., \& Valdettaro, L. 2010, J. Fluid Mech., 643, 363

Storch, N. I., \& Lai, D. 2014, MNRAS, 438, 1526

Tobie, G., Mocquet, A., \& Sotin, C. 2005, Icarus, 177, 534

Wu, Y. 2005, ApJ, 635, 674 